\title{
Modular Multiplicative Divisor Labeling of Some Path Related Graphs
}

\author{
R. Revathi and R. Rajeswari* \\ Department of Mathematics, Sathyabama University, Chennai 600119; \\ revathi_kumaran@yahoo.com, rajeswarivel@yahoo.in
}

\begin{abstract}
A graph $\mathrm{G}(\mathrm{VE})$ with $|\mathrm{V}|=\mathrm{n}$ is said to have modular multiplicative divisor labeling if there exist a bijection $\mathrm{f:} \mathrm{V}(\mathrm{G}) \rightarrow$ $\{1,2, \ldots$,$\} and the induced function \mathrm{f}^{*}: \mathrm{E}(\mathrm{G}) \rightarrow\{0,1,2, \ldots, \mathrm{n}-1\}$ where $\mathrm{f}^{*}(\mathrm{uv})=\mathrm{f}(\mathrm{u}) \mathrm{f}(\mathrm{v})(\bmod \mathrm{n})$ such that $\mathrm{n}$ divides the sum of all edge labels of $\mathrm{G}$. We prove that the path $\mathrm{P}_{\mathrm{n}}$, and the graph $\mathrm{P}_{\mathrm{a}, \mathrm{b}}$ (a graph which connects two vertices by means of " $\mathrm{b}$ " internally disjoint paths of length "a" each), shadow graph of a path and the cartesian product $\mathrm{P}_{\mathrm{n}} \times \mathrm{P}_{1}$, (n is not a multiple of 6) admits modular multiplicative divisor labeling. Also we discuss the upper bound for the number of edges in a modular multiplicative divisor graphs.
\end{abstract}

AMS Subject Classification: $05 \mathrm{C78}$

Keywords: Graph Labeling, Path, Graph $\mathrm{P}_{\mathrm{a}, \mathrm{b}^{\mathrm{b}}}$, Shadow Graph $\mathrm{D}_{2}(\mathrm{G})$, The Cartesian Product $\mathrm{P}_{\mathrm{n}} \times \mathrm{P}_{1}$

\section{Introduction}

For all terminology and notation in graph theory we follow Harary ${ }^{4}$. In this paper we consider only finite, simple, connected and undirected graphs. By a labeling we mean a one-to-one mapping that carries a set of graph elements on to a set of numbers, called labels (usually the set of integers). Graph labelings were first introduced in the mid sixties. Labeled graphs are becoming an increasingly useful family of mathematical models for a broad range of applications. The qualitative labelings of graph elements have inspired research in diverse fields of human enquiry such as conflict resolution in social psychology, electrical circuit theory and energy crisis. Quantitative labelings of graphs have applications in coding theory problems including the design of good radar location nodes, missile guidance codes etc. An enormous body of literature has grown around the subject, especially in the last thirty five years and is still getting embellished due to increasing number of application driven concepts ${ }^{5}$.
Also graph labeling is a strong communication between number theory ${ }^{2}$ and structure of graphs ${ }^{1}$. Modular multiplication plays an important role in number theory problems ${ }^{2}$. Through some mathematical logic we could able to introduce a new labeling called modular multiplicative divisor (MMD) labeling and we proved ${ }^{3}$ complete graph $\mathrm{K}_{\mathrm{n}}$, for all prime number $\mathrm{n}>3$, complete bipartite graph $\mathrm{K}_{\mathrm{m}, \mathrm{n}}$, cycle graph $\mathrm{C}_{\mathrm{n}}, \mathrm{n} \equiv 1,2(\bmod 3)$ are modular multiplicative divisor graphs. Also we proved ${ }^{9}$ split graph of cycle $\mathrm{C}_{\mathrm{n}}$, helm graph $\mathrm{H}_{\mathrm{n}}$, flower graph $\mathrm{f}_{\mathrm{n} \times 4}$, cycle cactus $\mathrm{C}_{4(\mathrm{n})}$, extended triplicate graph of a path are modular multiplicative divisor graphs. Modular multiplicative divisor labeling techniques can be applied in the field of cryptography ${ }^{7}$. A pattern of ordering edge labels can be taken as the secret key in hashing the plain text for Authentication of the message. The present work is intended to discuss the existence of MMD labeling of a path and path related graphs and the maximum number of edges in a modular multiplicative divisor graphs.

*Author for correspondence 


\section{Definitions Related to the Context}

\section{DEFINITION 2.1}

A graph labeling is an assignment of integers to the vertices or edges, or both, subject to certain conditions.

\section{DEFINITION 2.2}

A path in a graph is a sequence of vertices and edges, beginning and ending with vertices, such that each edge is incident with the vertices preceding and following it. Edges and vertices appear only once in a path.

\section{DEFINITION 2.3}

The shadow graph $D_{2}(G)$ of a connected graph $G$ is constructed by taking two copies of $G$ say $G^{\prime}$ and $G^{\prime \prime}$ join each vertex $v^{\prime}$ in $G^{\prime}$ to the neighbours of the corresponding vertex v" in $\mathrm{G}^{\prime \prime}$.

\section{DEFINITION 2.4}

Let $\mathrm{u}$ and $\mathrm{v}$ be two vertices. Connect $\mathrm{u}$ and $\mathrm{v}$ by means of " $b$ " internally disjoint paths of length "a" each. The resulting graph is denoted by $\mathbf{P}_{\mathrm{a}, \mathrm{b}}$.

\section{DEFINITION 2.5}

The cartesian product of two path graphs, one of which has only one edge is $\mathrm{P}_{\mathrm{n}} \times \mathrm{P}_{1}$. It is called ladder graph $\mathrm{L}_{\mathrm{n}}$. It is a planar undirected graph with $2 \mathrm{n}$ vertices and $n+2(n-1)$ edges.

\section{Discussion of MMD Labeling of Path Related Graphs}

In this section we consider the problem of whether the graphs in certain well-known and much-studied related graphs are modular multiplicative divisor. Here the discussion of MMD labeling of graphs under consideration is path and path related graphs (obtained through some graph operations).

\section{THEOREM: 3.1}

Path $\mathrm{P}_{\mathrm{n}}, \mathrm{n}>1$ admits modular multiplicative divisor labeling.

\section{Proof:}

Let $P_{n}$ be the path of $n$ vertices $\left\{v_{1}, v_{2}, v_{3}, \ldots, v_{n}\right\}$ and $n-1$ edges $\left\{e_{1}, e_{2}, e_{3}, \ldots e_{n-1}\right\}$.
Define a bijection $\mathrm{f}: \mathrm{V}(\mathrm{G}) \rightarrow\{1,2, \ldots, \mathrm{n}\}$ as follows. Case (i) Let $n=4 t+2, t=0,1,2,3, \ldots$.

$$
\begin{aligned}
& \mathrm{f}\left(\mathrm{v}_{\mathrm{n}-4}\right)=\frac{3 n-2}{4}, \mathrm{n}>2 \\
& \mathrm{f}\left(\mathrm{v}_{4 \mathrm{i}+1}\right)=\mathrm{i}+1,0 \leq \mathrm{i} \leq \frac{n-2}{4} \\
& \mathrm{f}\left(\mathrm{v}_{4 \mathrm{i}+3}\right)=\mathrm{n}-\mathrm{i}-1,0 \leq \mathrm{i} \leq \frac{n-6}{4}, \mathrm{n}>2
\end{aligned}
$$

Sub case $(\mathbf{i})$ when $t=0,2,4, \ldots$

$$
\begin{aligned}
\mathrm{f}\left(\mathrm{v}_{\mathrm{n}}\right) & =\mathrm{n} \\
\mathrm{f}\left(\mathrm{v}_{8 \mathrm{i}-4}\right) & =\frac{n+2}{4}+\mathrm{i}, 1 \leq \mathrm{i} \leq \frac{n-2}{8}, \mathrm{n}>2 \\
\mathrm{f}\left(\mathrm{v}_{8 \mathrm{i}}\right) & =\mathrm{n}-\frac{n+2}{4}-\mathrm{i}, 1 \leq \mathrm{i} \leq \frac{n-2}{8}, \mathrm{n}>2 \\
\mathrm{f}\left(\mathrm{v}_{4 \mathrm{i}-2}\right) & =\frac{3 n+2}{8}+\mathrm{i}, 1 \leq \mathrm{i} \leq \frac{n-6}{4}, \mathrm{n}>2
\end{aligned}
$$

Sub case $($ ii) when $t=1,3,5, \ldots$

$$
\begin{aligned}
& \mathrm{f}\left(\mathrm{v}_{\mathrm{n}}\right)=\frac{n}{2} ; \mathrm{f}\left(\mathrm{v}_{\mathrm{n}-2}\right)=\mathrm{n} ; \\
& \mathrm{f}\left(\mathrm{v}_{8 \mathrm{i}-4}\right)=\frac{n+2}{4}+\mathrm{i}, 1 \leq \mathrm{i} \leq \frac{n-6}{8}, \mathrm{n}>6 \\
& \mathrm{f}\left(\mathrm{v}_{8 \mathrm{i}}\right)=\mathrm{n}-\frac{n+2}{4}-\mathrm{i}, 1 \leq \mathrm{i} \leq \frac{n-6}{8}, \mathrm{n}>6 \\
& \mathrm{f}\left(\mathrm{v}_{4 \mathrm{i}-2}\right)=\left\{\begin{array}{l}
\frac{3 n-2}{8}+i, 1 \leq i \leq \frac{n-6}{8}, \mathrm{n}>6 \\
\frac{3 n-2}{8}+1+i, \frac{n+2}{8} \leq i \leq \frac{n-6}{4}, n>6
\end{array}\right.
\end{aligned}
$$

Case (ii): Let $\mathrm{n}=4 \mathrm{t}+3, \mathrm{t}=0,1,2,3, \ldots$

$$
\begin{aligned}
& \mathrm{f}\left(\mathrm{v}_{4 i+1}\right)=\mathrm{i}+1,0 \leq \mathrm{i} \leq \frac{n-3}{4} \\
& \mathrm{f}\left(\mathrm{v}_{4 \mathrm{ii}+3}\right)=\mathrm{n}-\mathrm{i}-1,0 \leq \mathrm{i} \leq \frac{n-3}{4}
\end{aligned}
$$

Sub case $(i)$ when $t=0,2,4, \ldots$

$$
\begin{aligned}
\mathrm{f}\left(\mathrm{v}_{\mathrm{n}-1}\right) & =\mathrm{n} ; \mathrm{f}\left(\mathrm{v}_{2+4 \mathrm{i}}\right)=\frac{3 n+7}{8}+\mathrm{i}, 0 \leq \mathrm{i} \leq \frac{n-7}{4}, \mathrm{n}>3 \\
\mathrm{f}\left(\mathrm{v}_{8 \mathrm{i}-4}\right) & =\frac{n+1}{4}+\mathrm{i}, 1 \leq \mathrm{i} \leq \frac{n-3}{8}, \mathrm{n}>3 \\
\mathrm{f}\left(\mathrm{v}_{8 \mathrm{i}}\right) & =\mathrm{n}-\frac{n+1}{4}-\mathrm{i}, 1 \leq \mathrm{i} \leq \frac{n-3}{8}, \mathrm{n}>3
\end{aligned}
$$


Sub case (ii) when $t=1,3,5, \ldots$.

$$
\begin{aligned}
\mathrm{f}\left(\mathrm{v}_{\mathrm{n}-3}\right) & =\mathrm{n} ; \mathrm{f}\left(\mathrm{v}_{2+4 \mathrm{i}}\right)=\frac{3(n+1)}{8}+\mathrm{i}, 0 \leq \mathrm{i} \leq \frac{n-3}{4} \\
\mathrm{f}\left(\mathrm{v}_{8 \mathrm{i}-4}\right) & =\frac{n+1}{4}+\mathrm{i}, 1 \leq \mathrm{i} \leq \frac{n-7}{8}, \mathrm{n}>7 \\
\mathrm{f}\left(\mathrm{v}_{8 \mathrm{i}}\right) & =\mathrm{n}-\frac{n+1}{4}-\mathrm{i}, 1 \leq \mathrm{i} \leq \frac{n-7}{8}, \mathrm{n}>7
\end{aligned}
$$

Case (iii): Let $\mathrm{n}=4 \mathrm{t}+4, \mathrm{t}=0,1,2,3, \ldots$

$$
\begin{aligned}
& \mathrm{f}\left(\mathrm{v}_{4 \mathrm{i}+1}\right)=\mathrm{i}+1,0 \leq \mathrm{i} \leq \frac{n-4}{4} \\
& \mathrm{f}\left(\mathrm{v}_{4 \mathrm{i}+3}\right)=\mathrm{n}-\mathrm{i}-1,0 \leq \mathrm{i} \leq \frac{n-4}{4}
\end{aligned}
$$

Sub case (i) when $t=0,2,4, \ldots$

$$
\begin{aligned}
\mathrm{f}\left(\mathrm{v}_{\mathrm{n}}\right) & =\mathrm{n} ; \mathrm{f}\left(\mathrm{v}_{2+4 \mathrm{i}}\right)=\frac{3 n+4}{8}+\mathrm{i}, 0 \leq \mathrm{i} \leq \frac{n-4}{4} \\
\mathrm{f}\left(\mathrm{v}_{8 \mathrm{i}-4}\right) & =\frac{n}{4}+\mathrm{i}, 1 \leq \mathrm{i} \leq \frac{n-4}{8}, \mathrm{n}>4 \\
\mathrm{f}\left(\mathrm{v}_{8 \mathrm{i}}\right) & =\mathrm{n}-\frac{n}{4}-\mathrm{i}, 1 \leq \mathrm{i} \leq \frac{n-4}{8}, \mathrm{n}>4
\end{aligned}
$$

Sub case (ii) when $\mathrm{t}=1,3,5, \ldots$.

$$
\begin{aligned}
& \mathrm{f}\left(\mathrm{v}_{\mathrm{n}}\right)=\frac{n}{2} ; \mathrm{f}\left(\mathrm{v}_{\mathrm{n}-4}\right)=\mathrm{n} \\
& \mathrm{f}\left(\mathrm{v}_{8 \mathrm{i}-4}\right)=\frac{n}{4}+\mathrm{i}, 1 \leq \mathrm{i} \leq \frac{n-8}{8}, \mathrm{n}>8 \\
& \mathrm{f}\left(\mathrm{v}_{8 \mathrm{i}}\right)=\mathrm{n}-\frac{n}{4}-\mathrm{i}, 1 \leq \mathrm{i} \leq \frac{n-8}{8}, \mathrm{n}>8 \\
& \mathrm{f}\left(\mathrm{v}_{2+4 \mathrm{i}}\right)=\left\{\begin{array}{l}
\frac{3 n}{8}+i, 0 \leq i \leq \frac{n-8}{8} \\
\frac{3 n}{8}+1+i, \frac{n}{8} \leq i \leq \frac{n-4}{4}
\end{array}\right.
\end{aligned}
$$

Case (iv) Let $\mathrm{n}=4 \mathrm{t}+5, \mathrm{t}=0,1,2,3, \ldots$

$$
\mathrm{f}\left(\mathrm{v}_{4 \mathrm{i}+3}\right)=\mathrm{n}-\mathrm{i}-1,0 \leq \mathrm{i} \leq \frac{n-5}{4}
$$

Sub case (i) when $t=0,2,4, \ldots$.

$$
\begin{array}{r}
\mathrm{f}\left(\mathrm{v}_{\mathrm{n}}\right)=\frac{3 n+1}{8} ; \mathrm{f}\left(\mathrm{v}_{\mathrm{n}-1}\right)=\mathrm{n} \\
\mathrm{f}\left(\mathrm{v}_{4 \mathrm{i}+1}\right)=\mathrm{i}+1,0 \leq \mathrm{i} \leq \frac{n-5}{4}
\end{array}
$$

$$
\begin{aligned}
& \mathrm{f}\left(\mathrm{v}_{4 i-2}\right)=\frac{3 n+1}{8}+\mathrm{i}, 1 \leq \mathrm{i} \leq \frac{n-1}{4} \\
& \mathrm{f}\left(\mathrm{v}_{8 \mathrm{i}-4}\right)=\frac{n-1}{4}+\mathrm{i}, 1 \leq \mathrm{i} \leq \frac{n-5}{8}, \mathrm{n}>5 \\
& \mathrm{f}\left(\mathrm{v}_{8 \mathrm{i}}\right)=\mathrm{n}-\frac{n-1}{4}-\mathrm{i}, 1 \leq \mathrm{i} \leq \frac{n-5}{8}, \mathrm{n}>5
\end{aligned}
$$

Sub case (ii) when $t=1,3,5, \ldots$.

$$
\begin{aligned}
\mathrm{f}\left(\mathrm{v}_{4 \mathrm{i}+1}\right) & =\mathrm{i}+1,0 \leq \mathrm{i} \leq \frac{n-1}{4} \\
\mathrm{f}\left(\mathrm{v}_{\mathrm{n}-7}\right) & =\frac{3 n-3}{4} ; \mathrm{f}\left(\mathrm{v}_{\mathrm{n}-3}\right)=\mathrm{n} ; \\
\mathrm{f}\left(\mathrm{v}_{8 \mathrm{i}-4}\right) & =\frac{n+3}{4}+\mathrm{i}, 1 \leq \mathrm{i} \leq \frac{n-1}{8} \\
\mathrm{f}\left(\mathrm{v}_{8 \mathrm{i}}\right) & =\mathrm{n}-\frac{n+3}{4}-\mathrm{i}, 1 \leq \mathrm{i} \leq \frac{n-1}{8}
\end{aligned}
$$

For $\mathrm{n}>9$

$$
\mathrm{f}\left(\mathrm{v}_{4 \mathrm{i}-2}\right)=\frac{3 n+5}{8}+\mathrm{i}, 1 \leq \mathrm{i} \leq \frac{n-9}{4}
$$

If the vertices are labeled as above and if we define an induced function $f^{*}: \mathrm{E}(\mathrm{G}) \rightarrow\{0,1,2, \ldots \mathrm{n}-1\}$ as $f^{\prime \prime}(u v)=f(u) f(v)$ mod $n$ then the sum of all edge labels is a multiple of $\mathrm{n}$. Hence the path $\mathrm{P}_{\mathrm{n}}, \mathrm{n}>1$ is a modular multiplicative divisor graph.

\section{THEOREM: 3.2}

The graph $\mathrm{P}_{2, \mathrm{~m}}$ admits MMD Labeling.

\section{ProOF:}

Let $\mathrm{P}_{2, \mathrm{~m}}$ be the graph obtained by connecting two vertices $\mathrm{u}$ and $\mathrm{v}$ by means of " $\mathrm{m}$ " internally disjoint paths of length "2" each. The resulting graph is denoted by $\mathrm{P}_{2, \mathrm{~m}}$.

Let $\mathrm{V}=\left\{\mathrm{u}, \mathrm{v}, \mathrm{v}_{1}, \mathrm{v}_{2}, \mathrm{v}_{3}, \ldots, \mathrm{v}_{\mathrm{m}}\right\}$ be the vertex set with $|V|=m+2=n$ (say) and $E=\left\{e_{1}, e_{2}, e_{3}, \ldots e_{2 m}\right\}$ be the edge set of the graph $\mathrm{P}_{2, \mathrm{~m}}$.

Define a bijection $\mathrm{f}: \mathrm{V}(\mathrm{G}) \rightarrow\{1,2, \ldots$,$\} and the induced$ function $\mathrm{f}^{\prime \prime}: \mathrm{E}(\mathrm{G}) \rightarrow\{0,1,2, \ldots \mathrm{n}-1\}$ where $\mathrm{f}^{\prime \prime}(\mathrm{uv})=\mathrm{f}(\mathrm{u})$ $\mathrm{f}(\mathrm{v}) \bmod \mathrm{n}$ as $\mathrm{f}(\mathrm{u})=1, \mathrm{f}(\mathrm{v})=\mathrm{n}-1, \mathrm{f}\left(\mathrm{v}_{\mathrm{m}}\right)=\mathrm{n}$ and $\mathrm{f}\left(\mathrm{v}_{\mathrm{i}}\right)=$ $\mathrm{i}+1$ for $1 \leq \mathrm{i} \leq \mathrm{m}-1$

Let the sum of all edge labels be $S$, then

$$
S=\sum_{i=1}^{m} f^{*}\left(w w_{i}\right)+\sum_{i=1}^{m} f^{*}\left(v v_{i}\right)
$$


We need to prove that $S$ is a multiple of $n$.

$$
\begin{aligned}
S= & {\left[\mathrm{f}(\mathrm{u}) \mathrm{f}\left(\mathrm{v}_{1}\right)+\mathrm{f}(\mathrm{u}) \mathrm{f}\left(\mathrm{v}_{2}\right)+\ldots+\mathrm{f}(\mathrm{u}) \mathrm{f}\left(\mathrm{v}_{\mathrm{m}}\right)+\mathrm{f}(\mathrm{v}) \mathrm{f}\left(\mathrm{v}_{1}\right)\right.} \\
& \left.+\mathrm{f}(\mathrm{v}) \mathrm{f}\left(\mathrm{v}_{2}\right)+\ldots+\mathrm{f}(\mathrm{v}) \mathrm{f}\left(\mathrm{v}_{\mathrm{m}}\right)\right] \bmod \mathrm{n} \\
= & {[\mathrm{f}(\mathrm{u})+\mathrm{f}(\mathrm{v})] \sum_{i=1}^{m} f\left(v_{i}\right) \bmod n } \\
= & n \sum_{i=1}^{m} f\left(v_{i}\right) \bmod n
\end{aligned}
$$

which is a multiple of $\mathrm{n}$. Hence the graph $\mathrm{P}_{2, \mathrm{~m}}$ admits modular multiplicative divisor labeling.

\section{EXAMPLE: $3 \cdot 3$}

The MMD labeling of the graph obtained by connecting two vertices $\mathrm{u}$ and $\mathrm{v}$ by means of 5 internally disjoint paths of length 2 each is shown in Figure 1.

\section{THEOREM: 3.4}

Shadow graph of a path $\mathrm{D}_{2}\left(\mathrm{P}_{\mathrm{n}}\right)$ admits modular multiplicative divisor labeling.

\section{ProOF:}

Let $P_{n}$ be the path with $n$ vertices and $n-1$ edges and $D_{2}\left(P_{n}\right)$ be the shadow graph of a path $P_{n}$ with $V=\left\{v_{1}{ }^{\prime}, v_{2}{ }^{\prime}\right.$, $\left.\mathrm{v}_{3}{ }^{\prime}, \ldots, \mathrm{v}_{\mathrm{n}}{ }^{\prime}, \mathrm{v}_{1}{ }^{\prime}, \mathrm{v}_{2}{ }^{\prime}, \mathrm{v}_{3}{ }^{\prime}, \ldots, \mathrm{v}_{\mathrm{n}}{ }^{\prime}\right\}$ as the vertex set and $\mathrm{E}=\mathrm{E}_{1}$ $\mathrm{UE}_{2} \mathrm{UE}_{3} \mathrm{UE}_{4}$ as the edge set where

$$
\begin{array}{ll}
\mathrm{E}_{1}=\left\{\mathrm{v}_{\mathrm{i}} \mathrm{v}_{\mathrm{i}+1}{ }^{\prime \prime}\right\} & 1 \leq \mathrm{i} \leq \mathrm{n}-1 \\
\mathrm{E}_{2}=\left\{\mathrm{v}_{\mathrm{i}+1}{ }^{\prime} \mathrm{v}_{\mathrm{i}}{ }^{\prime}\right\} & 1 \leq \mathrm{i} \leq \mathrm{n}-1 \\
\mathrm{E}_{3}=\left\{\mathrm{v}_{\mathrm{i}}{ }^{\prime} \mathrm{v}_{\mathrm{i}+1}{ }^{\prime}\right\} & 1 \leq \mathrm{i} \leq \mathrm{n}-1 \\
\mathrm{E}_{4}=\left\{\mathrm{v}_{\mathrm{i}}{ }^{\prime} \mathrm{v}_{\mathrm{i}+1}{ }^{\prime \prime}\right\} & 1 \leq \mathrm{i} \leq \mathrm{n}-1
\end{array}
$$

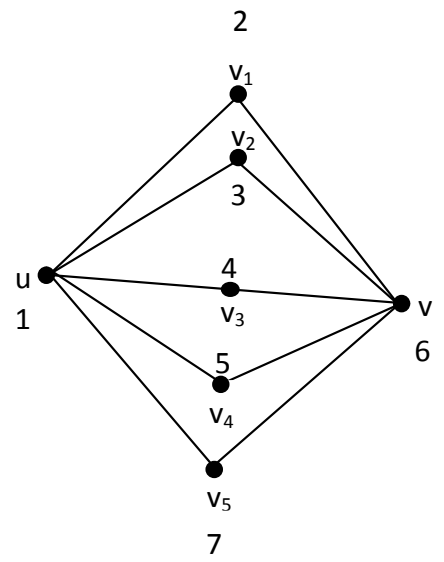

Figure 1. Modular Multiplicative Divisor Labeling For $\mathrm{P}_{2,5}$.
Define a bijection $\mathrm{f}: \mathrm{V}(\mathrm{G}) \rightarrow\{1,2, \ldots, 2 \mathrm{n}\}$ and the induced function $\mathrm{f}^{*}: \mathrm{E}(\mathrm{G}) \rightarrow\{0,1,2, \ldots 2 \mathrm{n}-1\}$ where $f^{*}(u v)=f(u) f(v) \bmod 2 n$ as

$$
\begin{aligned}
& \mathrm{f}\left(\mathrm{v}_{\mathrm{n}}^{\prime \prime}\right)=2 \mathrm{n} \\
& \mathrm{f}\left(\mathrm{v}_{\mathrm{i}}^{\prime}\right)=\mathrm{i} \quad 1 \leq \mathrm{i} \leq \mathrm{n} \\
& \mathrm{f}\left(\mathrm{v}_{\mathrm{i}}^{\prime \prime}\right)=2 \mathrm{n}-\mathrm{i} \quad 1 \leq \mathrm{i} \leq \mathrm{n}-1
\end{aligned}
$$

Let the sum of all edge labels be $S$, then

$$
\begin{aligned}
S= & \sum_{i=1}^{n-1}\left[f^{*}\left(v_{i}^{\prime} v_{i+1}^{\prime \prime}\right)+f^{*}\left(v_{i+1}{ }^{\prime} v_{i}^{\prime \prime}\right)\right. \\
& \left.+f^{*}\left(v_{i}^{\prime} v_{i+1}^{\prime}\right)+f^{*}\left(v_{i}^{\prime \prime} v_{i+1}^{\prime \prime}\right)\right]
\end{aligned}
$$

We need to prove that $S$ is a multiple of $2 n$.

$$
\begin{aligned}
S= & \sum_{i=1}^{n-1}\left[f\left(v_{i}^{\prime}\right) f\left(v_{i+1}^{\prime \prime}\right)+f\left(v_{i+1}^{\prime}\right) f\left(v_{i}^{\prime \prime}\right)+f\left(v_{i}^{\prime}\right) f\left(v_{i+1}^{\prime}\right)\right. \\
& \left.+f\left(v_{i}^{\prime \prime}\right) f\left(v_{i+1}^{\prime \prime}\right)\right] \text { mond } 2 n \\
= & \left\{\sum_{i=1}^{n-2}[i(2 n-1-i)+(2 n-i)(2 n-1-i)]\right. \\
& \left.+\sum_{i=1}^{n-1}[(i+1)(2 n-i)+i(i+1)]+(n-1) 2 n+2 n(n+1)\right\} \bmod 2 n
\end{aligned}
$$

which is a multiple of $2 n$.

Hence the shadow graph of a path $\mathrm{D}_{2}\left(\mathrm{P}_{\mathrm{n}}\right)$ admits Modular Multiplicative Divisor labeling.

\section{EXAMPLE: $3 \cdot 5$}

The MMD labeling for $\mathrm{D}_{2}\left(\mathrm{P}_{6}\right)$ is shown in Figure 2 .

\section{THEOREM: 3.6}

The Cartesian product $\mathrm{P}_{\mathrm{n}} \times \mathrm{P}_{1}$, ( $\mathrm{n}$ is not a multiple of 6) admits modular multiplicative divisor labeling.

\section{Proof:}

Case (i) when $\mathrm{n} \equiv 1(\bmod 2)$

Let $\mathrm{V}=\left\{\mathrm{v}_{1}, \mathrm{v}_{2}, \mathrm{v}_{3}, \ldots, \mathrm{v}_{\mathrm{n}}, \mathrm{v}_{\mathrm{n}+1}, \ldots, \mathrm{v}_{2 \mathrm{n}}\right\}$ be the vertex set and $E=E_{1} U E_{2} U E_{3}$ be the edge set where $E_{1}=\left\{v_{i} v_{i+1}\right.$,

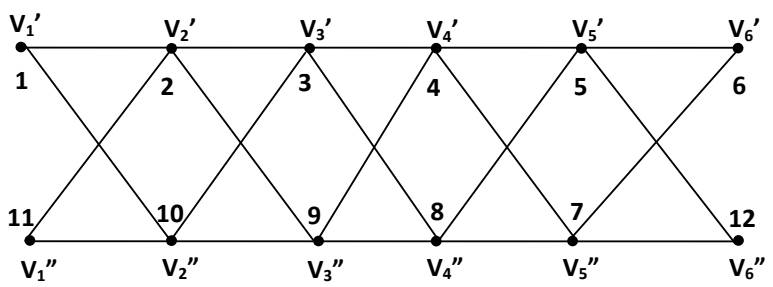

Figure 2. Modular multiplicative divisor labeling for $\mathrm{D}_{2}\left(\mathrm{P}_{6}\right)$. 
$1 \leq \mathrm{i}<\mathrm{n}\}, \mathrm{E}_{2}=\left\{\mathrm{v}_{\mathrm{i}} \mathrm{v}_{\mathrm{n}+\mathrm{i}}, 1 \leq \mathrm{i} \leq \mathrm{n}\right\}$ and $\mathrm{E}_{3}=\left\{\mathrm{v}_{\mathrm{i}} \mathrm{v}_{\mathrm{i}+1}, \mathrm{n}+1 \leq \mathrm{i}\right.$ $\leq 2 \mathrm{n}-1\}$

Define a bijection $\mathrm{f}: \mathrm{V} \rightarrow\{1,2, \ldots, 2 \mathrm{n}\}$ as $\mathrm{f}\left(\mathrm{v}_{\mathrm{i}}\right)=\mathrm{i}$, $1 \leq \mathrm{i} \leq 2 \mathrm{n}$ and an induced function $\mathrm{f}^{*}: \mathrm{E}(\mathrm{G}) \rightarrow\{0,1,2, \ldots$, $2 \mathrm{n}-1\}$ such that $\mathrm{f}^{\prime \prime}\left(\mathrm{v}_{\mathrm{i}} \mathrm{v}_{\mathrm{j}}\right)=\mathrm{f}\left(\mathrm{v}_{\mathrm{i}}\right) \mathrm{f}\left(\mathrm{v}_{\mathrm{j}}\right) \bmod 2 \mathrm{n}$ for all $\mathrm{v}_{\mathrm{i}} \mathrm{v}_{\mathrm{j}} \in \mathrm{E}$ and $\mathrm{v}_{\mathrm{i}}, \mathrm{v}_{\mathrm{j}} \in \mathrm{V}$ to prove that

$$
\sum_{i=1}^{n-1} f^{*}\left(v_{i} v_{i+1}\right)+\sum_{i=1}^{n} f^{*}\left(v_{i} v_{n+i}\right)+\sum_{i=n+1}^{2 n-1} f^{*}\left(v_{i} v_{i+1}\right) \text { is a }
$$

multiple of $2 \mathrm{n}$.

For the edges in $\mathrm{E}_{1}$ :

$$
\begin{aligned}
\sum_{i=1}^{n-1} f^{*}\left(v_{i} v_{i+1}\right) & =\sum_{i=1}^{n-1} f\left(v_{i}\right) f\left(v_{i+1}\right) \bmod 2 n \\
& =\left[\mathrm{n}\left(\mathrm{n}^{2}-1\right) / 3\right] \bmod 2 \mathrm{n}
\end{aligned}
$$

For the edges in $\mathrm{E}_{2}$ :

$$
\begin{aligned}
\sum_{i=1}^{n} f^{*}\left(v_{i} v_{n+i}\right) & =\sum_{i=1}^{n} f\left(v_{i}\right) f\left(v_{n+i}\right) \bmod 2 n \\
& =[\mathrm{n}(\mathrm{n}+1)(5 \mathrm{n}+1) / 6] \bmod 2 \mathrm{n}
\end{aligned}
$$

For the edges in $\mathrm{E}_{3}$ :

$$
\begin{aligned}
\sum_{i=n+1}^{2 n-1} f^{*}\left(v_{i} v_{i+1}\right) & =\sum_{i=n+1}^{2 n-1} f\left(v_{i}\right) f\left(v_{i+1}\right) \bmod 2 n \\
& =\left\{\mathrm{n}\left(13 \mathrm{n}^{2}-12 \mathrm{n}-1\right) / 3\right\} \bmod 2 \mathrm{n}
\end{aligned}
$$

Let $\mathrm{S}=$ Sum of all edge labels of $\mathrm{P}_{\mathrm{n}} \times \mathrm{P}_{1}, \mathrm{n}$ is odd, then $S=\left[n\left(11 n^{2}-6 n-1\right) / 2\right] \bmod 2 n$

When $\mathrm{n}$ is odd say $2 \mathrm{k}+1$

$\mathrm{S}=2(2 \mathrm{k}+1)\left(11 \mathrm{k}^{2}+8 \mathrm{k}+1\right)$ which is a multiple of $2 \mathrm{n}$.

Case (ii) when $n$ is even and not a multiple of 6 .

Let $\mathrm{V}=\left\{\mathrm{v}_{1}, \mathrm{v}_{2}, \mathrm{v}_{3}, \ldots, \mathrm{v}_{\mathrm{n}}, \mathrm{v}_{\mathrm{n}+1}, \ldots, \mathrm{v}_{2 \mathrm{n}}\right\}$ be the vertex set and $\mathrm{E}=\mathrm{E}_{1} \mathrm{UE}_{2}$ be the edge set where $\mathrm{E}_{1}=\left\{\mathrm{v}_{\mathrm{i}} \mathrm{v}_{\mathrm{i}+1}, 1 \leq \mathrm{i} \leq\right.$ $2 \mathrm{n}-1\}$ and $\mathrm{E}_{2}=\left\{\mathrm{v}_{\mathrm{i}} \mathrm{v}_{2 \mathrm{n}+1-\mathrm{i}}, 1 \leq \mathrm{i} \leq \mathrm{n}\right\}$.

Define a bijection $\mathrm{f}: \mathrm{V} \rightarrow\{1,2, \ldots, 2 \mathrm{n}\}$ as $\mathrm{f}\left(\mathrm{v}_{\mathrm{i}}\right)=\mathrm{i}, 1 \leq \mathrm{i} \leq$ $2 \mathrm{n}$ and an induced function

$\mathrm{f}^{*}: \mathrm{E}(\mathrm{G}) \rightarrow\{0,1,2, \ldots, 2 \mathrm{n}-1\}$ such that $\mathrm{f}^{*}\left(\mathrm{v}_{\mathrm{i}} \mathrm{v}_{\mathrm{j}}\right)=\mathrm{f}\left(\mathrm{v}_{\mathrm{i}}\right)$ $\mathrm{f}\left(\mathrm{v}_{\mathrm{j}}\right) \bmod 2 \mathrm{n}$ for all $\mathrm{v}_{\mathrm{i}} \mathrm{v}_{\mathrm{j}} \in \mathrm{E}$ and $\mathrm{v}_{\mathrm{i}}, \mathrm{v}_{\mathrm{j}} \in \mathrm{V}$.

We need to prove that

$$
\sum_{i=1}^{2 n-1} f^{*}\left(v_{i} v_{i+1}\right)+\sum_{i=1}^{n-1} f^{*}\left(v_{i} v_{2 n+1-i}\right) \text { is a multiple of } 2 \mathrm{n} .
$$

For the edges in $\mathrm{E}_{1}$ :

$$
\begin{aligned}
\sum_{i=1}^{2 n-1} f^{*}\left(v_{i} v_{i+1}\right) & =\sum_{i=1}^{2 n-1} f\left(v_{i}\right) f\left(v_{i+1}\right) \bmod 2 n \\
& =\left[2 \mathrm{n}\left(4 \mathrm{n}^{2}-1\right) / 3\right] \bmod 2 \mathrm{n}
\end{aligned}
$$

For the edges in $\mathrm{E}_{2}$ :

$$
\begin{aligned}
\sum_{i=1}^{n-1} f^{*}\left(v_{i} v_{2 n+1-i}\right) & =\sum_{i=1}^{n-1} f\left(v_{i}\right) f\left(v_{2 n+1-i}\right) \bmod 2 n \\
& =\left[2 \mathrm{n}\left(\mathrm{n}^{2}-1\right) / 3\right] \bmod 2 \mathrm{n}
\end{aligned}
$$

Let $\mathrm{S}=$ Sum of all edge labels of $\mathrm{P}_{\mathrm{n}} \times \mathrm{P}_{1}, \mathrm{n}$ is even and not a multiple of 6 say $3(2 k+1) \pm 1$. Then $S$ is a multiple of $2 n$.

EXAMPLE: 3.7

The MMD labeling for $\mathrm{P}_{7} \times \mathrm{P}_{1}, \mathrm{P}_{8} \times \mathrm{P}_{1}$ is shown in Figure $3 \& 4$.

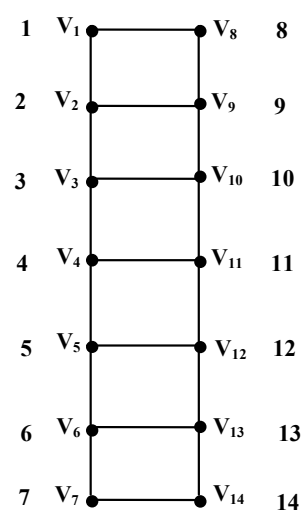

Figure 3. Modular multiplicative divisor labeling for $\mathrm{P}_{7} \times \mathrm{P}_{1}$.

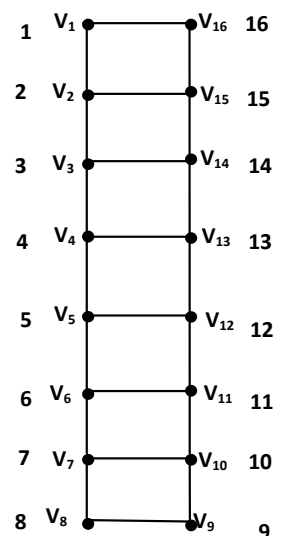

Figure 4. Modular multiplicative divisor labeling for $\mathrm{P}_{8} \times \mathrm{P}_{1}$. 


\section{Upper Bound for Number of Edges of a Modular Multiplicative Divisor Graphs}

In this section we study the number of edges in a modular multiplicative divisor graphs. Let $\lambda(\mathrm{n})$ be the maximum the number of edges in a modular multiplicative divisor graph of order $n$. We can find $\lambda(n)$ from the labeling of Complete graph $\mathrm{K}_{\mathrm{n}}$. Well-known result states that ${ }^{6}$ maximum number of edges in a simple graph of order $n$ is $\mathrm{n}(\mathrm{n}-1) / 2$. We proved that ${ }^{3}$ Complete graph $\mathrm{K}_{\mathrm{n}}$, for all prime number $\mathrm{n}>3$ admits MMD labeling. It follows from the result that maximum the number of edges in a modular multiplicative divisor graph of order $n>3$, a prime number is $n(n-1) / 2$. We put these facts into a theorem.

\section{THEOREM: 4.1}

The maximum number of edges $\lambda(\mathrm{n})$ in a modular multiplicative divisor graph of order $\mathrm{n}>3$, a prime number is $n(n-1) / 2$.

Further we improve the above result by giving relaxation for the restriction to $\mathrm{n}>3$, a prime number. $\mathrm{We}^{3}$ prove that the sum of all edge labels of a Complete graph $\mathrm{K}_{\mathrm{n}}$ for all prime number $\mathrm{n}>3$, is $\mathrm{n}(\mathrm{n}-1)(\mathrm{n}+1)$ $(3 n+2) / 24$, which is unique. If we relax $n>3$, a prime number to $\mathrm{n} \equiv 1(\bmod 2)$ as well as $\mathrm{n} \equiv 1,2(\bmod 3)$, we have $(\mathrm{n}-1)(\mathrm{n}+1) \equiv 0(\bmod 24)^{8}$. Hence we get modular multiplicative divisor graphs of order $n$, not only for prime numbers but also for composite numbers like $5^{2}$, $7^{2}, 11^{2}, 13^{2}, \ldots, 5^{3}, 7^{3}, 11^{3}, 13^{3}, \ldots\left(\right.$ i.e $\mathrm{n}^{\mathrm{m}} \equiv 1(\bmod 24)$, $\mathrm{m} \in \mathrm{N}-\{1\})$ and $35,55,65,77, \ldots$ admits MMD labeling. We conclude that the maximum number of edges $\lambda(n)$ in a modular multiplicative divisor graph of order $n>3$, $\mathrm{n} \equiv 1(\bmod 2)$ and $\mathrm{n} \equiv 1,2(\bmod 3)$ is $\mathrm{n}(\mathrm{n}-1) / 2$. From this study we get the following theorem.

\section{THEOREM: 4.2}

The maximum number of edges $\lambda(\mathrm{n})$ in a modular multiplicative divisor graph of order $\mathrm{n}>3, \mathrm{n} \equiv 1(\bmod 2)$ and $\mathrm{n} \equiv 1,2(\bmod 3)$ is $\mathrm{n}(\mathrm{n}-1) / 2$.

\section{Conclusion}

We prove that some path related graphs admits modular multiplicative divisor labeling and also studied the upper bound for the number of edges in a modular multiplicative divisor graphs of order $\mathrm{n}>3, \mathrm{n} \equiv 1(\bmod 2)$ and $\mathrm{n} \equiv 1,2(\bmod 3)$. We investigated the upper bound for number of edges $\lambda(n)$ in a modular multiplicative divisor graph of order $\mathrm{n}>3$, for $\mathrm{n} \equiv 0(\bmod 2)$ and $\mathrm{n} \equiv 3(\bmod 6)$.

\section{Acknowledgement}

We are very much thankful to Mr. Nagarajan, Assistant Professor, Department of CSE, Sathyabama University for his valuable suggestions and guidance in the field of Cryptography while the work was in progress.

\section{References}

1. Beineke LW, Hedge SM. Strongly Multiplicative Graph, Discuss, math. Graph Theory. 2001; 21:63-75.

2. Burton DM. Elementary Number Theory. 2nd ed. Wm. C. Brown Company Publishers; 1980.

3. Revathi R, Rajeswari R. On Modular Multiplicative Divisor Labeling of Zero Divisor Graphs. Proceedings of National conference on Mathematical Techniques and its Applications; 2012; p. 296-301.

4. Harary F. Graph Theory. Massachusetts: Addison-Wesley, Reading; 1972.

5. Gallian JA. A Dynamic Survey of Graph Labeling. The Electronic journal of combinatorics. 2010.

6. Deo N. Graph Theory with Applications to Engineering and Computer Science.

7. Stallings W. Cryptography and Network Security. New Delhi: Pearson/Prentice Hall; 2006.

8. Stein W. Elementary Number Theory. New York, LLC: Springer Science + Business Media; 2009.

9. Revathi R, Rajeswari R. On Modular Multiplicative Divisor Graphs. IEEE Proceedings of International Conference on Pattern Recognition, Informatics and Mobile Engineering (PRIME2013). 